\title{
Subdirect irreducibility of algebras and acts with an additional unary operation
}

\author{
N.V. Loi and R. Wiegandt
}




\title{
SUBDIRECT IRREDUCIBILITY OF ALGEBRAS AND ACTS WITH AN ADDITIONAL UNARY OPERATION
}

\author{
N. V. LOI AND R. WIEGANDT
}

[Received: December 12, 2004]

\begin{abstract}
Aвstract. As a generalization of algebras with involution [1], we consider algebras $(A ; \mathcal{F} \cup\{\varphi\})$ with an additional unary operation $\varphi$ of finite degree $k$. A subdirectly irreducible algebra $(A ; \mathcal{F} \cup\{\varphi\})$ is a subdirect product of subdirectly irreducible algebras $(A / \varphi(\Theta) ; \mathcal{F}), \ldots,\left(A / \varphi^{d}(\Theta) ; \mathcal{F}\right)$ where $\Theta$ is a certain congruence, $d$ is a divisor of $k$ and $\varphi^{d}$ is the identical mapping. For a congruence modular algebra $(A ; \mathcal{F})$ also the unique smallest congruence of $(A ; \mathcal{F} \cup\{\varphi\})$ described. Examples are $S$-acts with an additional unary operation. In this case more information is given on the subdirectly irreducible components.
\end{abstract}

Mathematics Subject Classification: 08A05, 08A60

Keywords: subdirectly irreducible algebra, congruence, unary operation, $S$-act

\section{Preliminaries}

The purpose of this note is to generalize the notion of involution of universal algebras and to study the structure of subdirectly irreducible universal algebras with generalized involution, that is, with an additional unary operation of finite degree.

Let $\mathbb{V}_{\varphi}$ be a variety of universal algebras $(A ; \mathcal{F} \cup\{\varphi\})$ with finitary operations $f \in \mathcal{F}$ and a unary operation $\varphi: a \rightarrow \varphi(a)$ subject to the identities

(i) $\left.E_{\lambda}=E_{\lambda}\left(f_{\lambda_{1}}, \ldots, f_{\lambda n_{\lambda}}\right) \mid f_{\lambda_{1}}, \ldots, f_{\lambda n_{\lambda}} \in \mathcal{F}\right), \lambda \in \Lambda$, involving only operations from $\mathcal{F}$,

(ii) $E_{\varphi}: \varphi^{k}(a)=a$,

where $k \geq 2$ is a fixed integer, the degree of $\varphi$,

(iii) $E_{f}: \varphi\left(f\left(a_{1}, \ldots, a_{n}\right)\right)=f\left(\varphi\left(a_{\pi_{f}(1)}, \ldots, \varphi\left(a_{\pi_{f}(n)}\right)\right)\right)$ for all $f \in \mathcal{F}$,

where $\pi_{f}$ is a permutation of $n$ elements ( $n$ the arity of the operation $f$ ) assigned to each operation $f \in \mathcal{F}$.

Each permutation $\pi_{f}$ can be written into a product of disjoint cyclic permutations $c\left(\pi_{f}\right)_{1}, \ldots, c\left(\pi_{f}\right)_{r}$ admitting also cyclic permutations of length 1 .

These identities may induce some further ones. The identity $f=\varphi^{k}(f)$ is a consequence of (ii), and this is not trivial if and only if the degree $k$ of $\varphi$ is not a multiple

Research supported by OTKA, Grant No. T043034. 
of the lengths $c\left(\pi_{f}\right)_{1}, \ldots, c\left(\pi_{f}\right)_{r}$. The identity $f=\varphi^{k}(f)$ may be viewed as a generalized commutativity; in the case of a binary operation $f\left(x_{1}, x_{2}\right)$, cyclic permutation $c\left(\pi_{f}\right)=(12)=\pi_{f}$ and odd degree $k=2 \ell+1$ we have

$$
f\left(x_{1}, x_{2}\right)=\varphi^{k}\left(f\left(x_{1}, x_{2}\right)\right)=\varphi^{2 \ell+1}\left(f\left(x_{1}, x_{2}\right)\right)=\varphi\left(f\left(x_{1}, x_{2}\right)\right)=f\left(x_{2}, x_{1}\right),
$$

that is, the commutativity of the operation $f$.

Moreover, if $f: \rightarrow a$ and $g: a \rightarrow g(a)$ are 0 -ary and unary operations, then by (iii) it follows that

$$
\varphi(f): \rightarrow a \quad \text { and } \quad \varphi(g(a))=g(\varphi(a)) .
$$

In particular, for the zero element 0 and the unity element 1 (if they exist) we have

$$
\varphi(0)=0 \quad \text { and } \quad \varphi(1)=1,
$$

and also

$$
\varphi(-a)=-\varphi(a)
$$

for the additive inverse of an element $a$.

We are going to scrutinize the interrelations between the variety $\mathbb{V}_{\varphi}$ and the variety $\mathbb{V}$ of algebras $(A ; \mathcal{F})$ subject to the identities (i).

Let us observe that for any algebra $(A ; \mathcal{F} \cup\{\varphi\})$ and congruence $\Theta \in \operatorname{Con}(A ; \mathcal{F})$ of the algebra $(A ; \mathcal{F}) \in \mathbb{V}$ also

$$
\varphi(\Theta)=\{(\varphi(a), \varphi(b)) \mid(a, b) \in \Theta\}
$$

is a congruence of $(A ; \mathcal{F})$, as seen readily from (iii).

Proposition 1. (a) Let $(A ; \mathcal{F} \cup\{\varphi\}) \in \mathbb{V}_{\varphi}$ and $\Theta \in \operatorname{Con}(A ; \mathcal{F})$. Then $\Theta=\varphi(\Theta)$ if and only if $\Theta \in \operatorname{Con}(A ; \mathcal{F} \cup\{\varphi\})$.

(b) The lattice $\operatorname{Con}(A ; \mathcal{F} \cup\{\varphi\})$ is a sublattice of the lattice $\operatorname{Con}(A ; \mathcal{F})$.

(c) If $\Theta \in \operatorname{Con}(A ; \mathcal{F})$, then $\varphi(\Theta) \vee \cdots \vee \varphi^{k}(\Theta)$ and $\varphi(\Theta) \wedge \cdots \wedge \varphi^{k}(\Theta)$ belong to $\operatorname{Con}(A ; \mathcal{F} \cup\{\varphi\})$.

Proof. The statements correspond to those of [1, Proposition 3.1] and their proofs are straightforward.

As a motivation for the subsequent investigations we give an example.

Example. Let $G$ be an abelian group, and consider the direct sum $A=G \oplus G$. On $A$ we define a unary operation $\varphi$ by

$$
\varphi(a, b)=(b, a+b) \quad(\forall a, b \in G) .
$$

Since

$$
\varphi((a, b)+(c, d))=(b+d, a+b+c+d)=\varphi(a, b)+\varphi(c, d),
$$

the operation fulfils the requirement of condition (iii). One readily sees that if $A$ is an elementary 2-group, then

$$
\varphi^{3}(a, b)=(a+2 b, 2 a+3 b)=(a, b),
$$


so the degree of $\varphi$ is 3 . Clearly, $(0, G)$ defines a congruence of $(A ;+)$,

$$
\varphi(0, G)=\{(a, a) \mid a \in G\}
$$

gives the diagonal congruence of $A$ and $\varphi^{2}(0, G)$ is the congruence determined by $(G, 0)$. If $|G|=2$, then all the three factors of $(A ;+)$ by these congruences are subdirectly irreducible, and $(A ;+)$ is a subdirect product of these factors. Nevertheless, $(A ;\{+, \varphi\})$ itself is subdirectly irreducible.

For elementary 3-,5-,7-,11- groups, the degree of $\varphi$ is $8,20,16,10$, respectively. If $G$ is a cyclic group of 4 elements or a direct sum of such groups, then the degree of $\varphi$ is 12 .

\section{SubdiRECT DECOMPOSITIONS}

As seen in [1], a subdirectly irreducible algebra $(A ; \mathcal{F} \cup\{\varphi\})$ with involution $\varphi$ (that is the degree of $\varphi$ is $k=2)$ is either subdirectly irreducible as an algebra $(A ; \mathcal{F}) \in \mathbb{V}$ or a certain subdirect product of two subdirectly irreducible algebras of $\mathbb{V}$. We shall generalize this result, and describe the subdirectly irreducible algebra $(A ; \mathcal{F} \cup\{\varphi\}) \in$ $\mathbb{V}_{\varphi}$ as a subdirect product of subdirectly irreducible algebras of $\mathbb{V}$ and see that the number $d$ of the subdirect components is a divisor of the degree $k$ of $\varphi$.

The smallest congruence of an act $A$ will be denoted by $\omega_{A}$ or briefly by $\omega$. We shall call the unique atom of the lattice of congruences of a subdirectly irreducible algebra the heart of that algebra. This terminology corresponds to that of ring theory: the unique atom in the lattice of ideals of a subdirectly irreducible ring used to be called the heart of that ring.

Theorem 2. If $(A ; \mathcal{F} \cup\{\varphi\})$ is a subdirectly irreducible algebra with heart $\chi_{\varphi}$, then there exists a congruence $\Theta \in \operatorname{Con}(A ; \mathcal{F})$ such that $\chi_{\varphi} \not \subset \Theta$ and $(A ; \mathcal{F})$ is isomorphic to a subdirect product of subdirectly irreducible algebras

$$
(A / \varphi(\Theta) ; \mathcal{F}),\left(A / \varphi^{2}(\Theta) ; \mathcal{F}\right), \ldots,\left(A / \varphi^{d}(\Theta) ; \mathcal{F}\right),
$$

where $d$ is the least positive integer such that $\varphi^{d}(\Theta)=\Theta$. The heart of $\left(A / \varphi^{i}(\Theta) ; \mathcal{F}\right)$ is $\chi_{i}=\left(\chi_{\varphi} \vee \varphi^{i}(\Theta)\right) / \varphi^{i}(\Theta)$ for each $i=1, \ldots, d$. The isomorphism between $(A ; \mathcal{F})$ and the subdirect product $\bigotimes_{i=1}^{d}\left(A / \varphi^{i}(\Theta) ; \mathcal{F}\right)$ is given by the correspondence

$$
a \longmapsto\left([a]_{\varphi(\Theta)}, \ldots,[a]_{\varphi^{d}(\Theta)}\right) \quad(\forall a \in A),
$$

where $[a]_{\varphi^{i}(\Theta)}$ stands for the $\varphi^{i}(\Theta)$-coset represented by $a \in A$.

Proof. If $(A ; \mathcal{F})$ is subdirectly irreducible, then $d=1$ and $\Theta=\omega=\varphi(\omega)=\varphi(\Theta)$ does the job.

Suppose, next, that $(A ; \mathcal{F})$ is not subdirectly irreducible. Since $\chi_{\varphi} \in \operatorname{Con}(A ; \mathcal{F})$, there exists a congruence $\Theta \in \operatorname{Con}(A ; \mathcal{F})$ such that $\omega \neq \Theta \neq \varphi(\Theta), \chi_{\varphi} \nsubseteq \Theta$ and by an appropriate choice of $\Theta$ it can be achieved (Zorn's Lemma) that $(A / \Theta ; \mathcal{F})$ is subdirectly irreducible and in this case the heart of $(A / \Theta ; \mathcal{F})$ is obviously $\chi_{1}=$ $\left(\chi_{\varphi} \vee \Theta\right) / \Theta$. Let $d$ be the smallest integer such that $\varphi^{d}(\Theta)=\Theta$. Such an integer $d$ 
does exist, and $d$ must divide $k$, the degree of $\varphi$. Moreover, by (ii) also $\left(A / \varphi^{i}(\Theta) ; \mathcal{F}\right)$ is subdirectly irreducible with heart $\chi_{i}$ for every $i=1, \ldots, d$. Since $\chi_{\varphi} \nsubseteq \Theta$ and $\varphi(\Theta) \wedge \varphi^{2}(\Theta) \wedge \cdots \wedge \varphi^{d}(\Theta)$ is in $\operatorname{Con}(A ; \mathcal{F} \cup\{\varphi\})$, we conclude that

$$
\varphi(\Theta) \wedge \varphi^{2}(\Theta) \wedge \cdots \wedge \varphi^{d}(\Theta)=\omega .
$$

Thus, $(A ; \mathcal{F})$ has the desired subdirect product representation.

The last assertion is obvious.

In the next two theorems we shall use the assumption that the congruence lattice of the algebra $(A ; \mathcal{F})$ is modular. This is always so for groups, rings, and in many other cases, but in general the congruence lattice of an algebra, in particular of an $S$-act, need not be modular.

Remark. In [1, Theorem 3.2] it has to be assumed that the congruence lattice of $(A ; \mathcal{F})$ is modular (this was used on line 8 of its proof). This inaccuracy, however, does not affect the further results of [1].

Theorem 3. Let $(A ; \mathcal{F} \cup\{\varphi\})$ be an algebra as in Theorem 2. If $(A ; \mathcal{F})$ is congruence modular and $\chi_{\varphi} \wedge \Theta=\omega$, then $\chi_{\varphi}$ is an atom in $\operatorname{Con}(A ; \mathcal{F})$ and there is a one-to-one correspondence between the $\chi_{\varphi}$-cosets of $(A ; \mathcal{F})$ and the $\chi_{i}$-cosets of $\left(A / \varphi^{i}(\Theta) ; \mathcal{F}\right)$ given by the correspondence

$$
[a]_{\chi_{\varphi}} \longmapsto\left[[a]_{\varphi^{i}(\Theta)}\right]_{\chi_{i}},
$$

which can be expressed also in terms of relations as

$$
a \chi_{\varphi} b \longmapsto[a]_{\varphi^{i}(\Theta)} \chi_{i}[b]_{\varphi^{i}(\Theta)} .
$$

Proof. If $d=1$, then there is nothing to prove.

Suppose that $d>1$, and let $\kappa \in \operatorname{Con}(A ; \mathcal{F})$ be a congruence such that $\kappa<\chi_{\varphi}$. Then either $\kappa \leq \Theta$ and $\kappa \leq \chi_{\varphi} \wedge \Theta=\omega$, or $\Theta<\kappa \vee \Theta$. In the latter case, taking into account that $\left(\chi_{\varphi} \vee \Theta\right) / \Theta$ is the heart of $(A / \Theta ; \mathcal{F})$, we have $\chi_{\varphi} \vee \Theta \leq \kappa \vee \Theta$ whence $\kappa \vee \Theta=\chi_{\varphi} \vee \Theta$. Now, the sublattice $\left\{\omega, \kappa, \chi_{\varphi}, \Theta, \chi_{\varphi} \vee \Theta\right\}$ is not modular unless $\kappa=\omega$. This proves that $\chi_{\varphi}$ is an atom in $\operatorname{Con}(A ; \mathcal{F})$.

In view of the relation $\chi_{\varphi} \wedge \Theta=\omega$, a moment's reflection shows the validity of the further statements.

Theorem 4. Let $(A ; \mathcal{F} \cup\{\varphi\})$ be an algebra as in Theorem 2. If $(A ; \mathcal{F})$ is congruence modular and $\chi_{\varphi} \wedge \Theta \neq \omega$, then

(1) $\bigwedge_{i \neq j=1}^{d}\left(\chi_{\varphi} \wedge \varphi^{j}(\Theta)\right)$ is an atom in $\operatorname{Con}(A ; \mathcal{F})$,

(2) $\bigvee_{i=1}^{d}\left(\bigwedge_{i \neq j=1}^{d}\left(\chi_{\varphi} \wedge \varphi^{j}(\Theta)\right)\right)=\chi_{\varphi}$

(3) $\bigwedge_{i \neq j=1}^{d}\left(\chi_{\varphi} \wedge \varphi^{j}(\Theta)\right) \wedge \bigvee_{i \neq j=1}^{d}\left(\bigwedge_{j \neq k=1}^{d}\left(\chi_{\varphi} \wedge \varphi^{k}(\Theta)\right)\right)=\omega$.

Proof. (1) It suffices to prove the statement for $i=d$. Since $A / \Theta$ is subdirectly irreducible with heart $\left(\chi_{\varphi} \vee \Theta\right) / \Theta$, the congruence $\chi_{\varphi} \wedge \Theta$ covers $\Theta$ in $\operatorname{Con}(A ; \mathcal{F})$. 
Let us consider a congruence $\kappa \in \operatorname{Con}(A ; \mathcal{F})$ such that $\chi_{\varphi} \wedge \Theta<\kappa \leq \chi_{\varphi}$. Then $\kappa \not \leq \Theta$ and by the subdirectly irreducibility of $A / \Theta$ we have

$$
\chi_{\varphi} \vee \Theta \leq \kappa \vee \Theta \leq \chi_{\varphi} \vee \Theta,
$$

that is, $\kappa \vee \Theta=\chi_{\varphi} \vee \Theta$. Thus by $\kappa \leq \chi_{\varphi}$ the modularity of $\operatorname{Con}(A ; \varphi)$ yields

$$
\chi_{\varphi}=\left(\chi_{\varphi} \vee \Theta\right) \wedge \chi_{\varphi}=(\kappa \vee \Theta) \wedge \chi_{\varphi}=\kappa \vee\left(\Theta \wedge \chi_{\varphi}\right)=\kappa,
$$

showing that $\chi_{\varphi}$ covers $\chi_{\varphi} \wedge \Theta$.

Next, we consider a congruence $\tau \in \operatorname{Con}(A ; \mathcal{F})$ such that

$$
\omega \neq \tau \leq \bigwedge_{i=1}^{d-1}\left(\chi_{\varphi} \wedge \varphi^{i}(\Theta)\right) .
$$

Since $\chi_{\varphi}$ covers $\chi_{\varphi} \wedge \Theta$, by $\tau \neq \omega=\bigwedge_{i=1}^{d}\left(\chi_{\varphi} \wedge \varphi^{i}(\Theta)\right)$ we have $\tau \vee\left(\chi_{\varphi} \wedge \Theta\right)=\chi_{\varphi}$. If $\tau \neq \bigwedge_{i \neq j=1}^{d}\left(\chi_{\varphi} \wedge \varphi^{j}(\Theta)\right)$ then $\left\{\omega, \tau, \bigwedge_{i \neq j=1}^{d}\left(\chi_{\varphi} \wedge \varphi^{j}(\Theta)\right), \chi_{\varphi} \wedge \Theta, \chi_{\varphi}\right\}$ is a non-modular sublattice in $\operatorname{Con}(A ; \mathcal{F})$. This contradiction proves the assertion in (1). holds.

(2) Since $\chi_{\varphi} \wedge \Theta \neq \omega$ and $\varphi\left(\bigvee_{i=1}^{d}\left(\chi_{\varphi} \wedge \varphi^{i}(\Theta)\right)\right)=\bigvee_{i=1}^{d}\left(\chi_{\varphi} \wedge \varphi^{i}(\Theta)\right)$, the assertion

(3) Assume that the expression in (3) is not equal $\omega$. Then, by (1), it equals $\bigwedge_{i \neq j=1}^{d}\left(\chi_{\varphi} \wedge \varphi^{j}(\Theta)\right)$, Hence by ii) we have

$$
\bigwedge_{i \neq j=1}^{d}\left(\chi_{\varphi} \wedge \varphi^{j}(\Theta)\right) \leq \bigvee_{i \neq j=1}^{d}\left(\bigwedge_{j \neq k=1}^{d}\left(\chi_{\varphi} \wedge \varphi^{k}(\Theta)\right)\right)=\chi_{\varphi} .
$$

This, however, contradicts $\varphi\left(\chi_{\varphi}\right)=\chi_{\varphi}$.

Restricting the considerations to an algebra $(A ; \mathcal{F})$ the congruences of which are determined by certain subalgebras (ideals, normal subgroups, etc.), then $(A ; \mathcal{F})$ is congruence modular. We shall use the language and notions of ring theory, and denote the unique minimal ideal (i. e., the heart) of $(A ; \mathcal{F} \cup\{\varphi\})$ by $H_{\varphi}$, and write $I$ for the ideal determined by the congrunce $\Theta$. Then Theorems 3 and 4 have the following meaning.

Corollary 5. If $H_{\varphi} \wedge I=0$, then $H_{\varphi}$ is an atom in the lattice of ideals of $(A ; \mathcal{F})$ and $H_{\varphi}$ is isomorphic to the heart $\left(H_{\varphi}+\varphi^{i}(I)\right) / \varphi^{i}(I)$ of $\left(A / \varphi^{i}(I) ; \mathcal{F}\right)$ for each $i=1, \ldots d$. If $H_{\varphi} \wedge I \neq 0$, then $\bigcap_{i \neq j=1}^{d}\left(H_{\varphi} \cap \varphi^{i}(I)\right)$ is an atom in the lattice of ideals of $(A ; \mathcal{F})$ for each $i=1, \ldots, d$, and $H_{\varphi}$ is isomorphic to the direct sum of these ideals.

\section{3. $S$-ACTS}

An $S$-act $A$ over a semigroup $S$ is a set $A$ with scalar multiplication $s a$ ( $s \in S$, $a \in A$ ) subject to the rule $s(t a)=(s t) a$. A one-element subact is called a sink. We call an $S$-act $A$ simple, if it has only two congruences: the all congruence $\iota_{A}$ and the 
equality congruence $\omega_{A}$. Recall that an $S$-act is strongly connected if $A=S a$ for every element $a \in A$. For details the reader may consult [2].

An $S$-act $A$ is, in fact, an algebra $(A ; S)$ where each element $s$ of the semigroup $S$ is a unary operation $s: a \mapsto s a, a \in A$, subject to the identity

$$
(s t) a=s(t a) \quad(\forall s, t \in S, \forall a \in A) .
$$

Having one more operation $\varphi$ on $A$ subject to conditions (ii) and (iii) of Sec. 1, the $(S, \varphi)$-act $(A ; S \cup\{\varphi\})$ satisfies the identity

$$
\varphi(s a)=s(\varphi(a) \quad(\forall s \in S, \forall a \in A),
$$

in other words, $\varphi$ is an automorphism of the $S$-act $A$ of order $k$.

Let $(A: S \cup\{\varphi\})$ be a subdirectly irreducible $(S, \varphi)$-act with heart $\chi_{\varphi}$, and $|A|>1$ Beside the decomposition Theorems 2, 3 and 4 we can say more on the structure of the $(S, \varphi)$-act $A$ in terms of the $S$-act $A$.

First, we make some obserations.

Let us consider the intersection $B=\cap\left(C_{\lambda} \mid \lambda \in \Lambda\right)$ of all $(S, \varphi)$-subacts $C_{\lambda}$ of $A$ with $|C|>1$. If the corresponding Rees congruence $\varrho_{B}$ to $B$ is $\omega$, then $A$ is not subdirectly irreducible, a contradiction. Hence $\chi_{\varphi} \leq \varrho_{B}$, and so $\chi_{\varrho}$ is a partition of $B$ and each element $a \in A \backslash B$ forms $a \chi_{\varphi}$-coset. The set $T$ of all $(S, \varphi)$-sinks of $A$ is an $(S, \varphi)$-subact, and so either $|T| \leq 1$ or $B \subseteq T$. Since $A$ is a subdirectly irreducible $(S, \varphi)$-act, the set $T$ cannot have more than 2 elements. By the minimality of $B$ in the latter case only $|B|=|T|=2$ is possible.

Thus, there exists a unique smallest $(S, \varphi)$-subact $B$ of $A$ with $|B|>1$, and one of the following cases may occur.

Case 1. $\chi_{\varphi}=\iota_{A}$.

$\chi_{\varphi} \neq \iota_{A}$ and

Case 2. There is no $(S, \varphi)$-sink in $B$.

Case 3. There is exactly one $(S, \varphi)$-sink in $B$.

Case 4. B consists of two $(S, \varphi)$-sinks.

Next, we discuss these cases.

Case 1. The subdirectly irreducible $(S, \varphi)$-act $A$ is simple. ¿From Theorem 2 we infer that $\Theta=\omega$. Since $\varphi(\omega)=\omega$, it follows that $d=1$ and that the $S$-act $A$ is subdirectly irrecducible by Theorem 3 . Thus, $A$ is a simple $S$-act which may possess one $S$-sink.

Case 2. Let $C$ be a non-empty $S$-subact of $B$. Since $B$ does not contain $(S, \varphi)$-sinks, necessarily

$$
C \cup \varphi(C) \cup \cdots \cup \varphi^{d-1}(C)=B
$$

and

$$
C \cap \varphi(C) \cap \cdots \cap \varphi^{d-1}(C)=\varnothing .
$$

Let $j_{1}$ be the least integer such that $C \cap \varphi^{j_{1}}(C) \neq \varnothing$. If such an integer $j_{1}$ does not exist, then $C$ is disjoint to each $\varphi^{i}(C)$ for every $i=1, \ldots, d-1$, and so is $\varphi^{i}(C)$ to each $\varphi^{j}(C)$ where $i \neq j \in\{1, \ldots, d\}$. Further, let $j_{2}$ be the next integer such that 
$C \cap \varphi^{j_{1}}(C) \cap \varphi^{j_{2}}(C) \neq \varnothing$, and so on. In this way we get integers $0<j_{1}<j_{2}<\cdots<$ $d-1$ such that

$$
D=C \cap \varphi^{j_{1}}(C) \cap \cdots \cap \varphi^{j_{r}}(C) \neq \varnothing,
$$

and the number $r$ is maximal. One readily sees that

$$
D \cap \varphi^{i}(D) \neq \varnothing \text { if } i=j_{1} \text { and } j_{2}=2 j_{1}, \ldots, j_{r}=r j_{1}, d=(r+1) j_{1} .
$$

By the same token we see that $D \cap \varphi^{i}(D) \neq \varnothing$ if and only if $i=j_{s}$ for some divisor $s$ of $d$. Now $D \cap \varphi^{i}(D)=\varnothing$ for every $i \notin\left\{j_{s}, 2 j_{2}, \ldots, d\right\}$. Hence $D, \varphi(D), \ldots, \varphi^{j_{1}-1}(D)$ are disjoint $S$-subacts. Since

$$
D=\varphi^{j_{1}}(D)=\varphi^{j_{2}}(D)=\cdots=\varphi^{j_{r}}(D),
$$

we conclude that $B$ is the disjoint union, that is, the coproduct

$$
B=D \amalg \varphi(D) \amalg \ldots \amalg \varphi^{j_{1}-1}(D) .
$$

By the minimality of $B$ the disjoint components $D, \varphi(D), \ldots, \varphi^{j_{1}-1}(D)$ are strongly connected $S$-acts. Observe that $\varphi^{j_{s}}(a) \in D$ for every $a \in D$, but $\varphi^{i}$ need not be the identical mapping. Thus, in Case 2 , the $(S, \varphi)$-subact $B$ is a coproduct of $j_{1} S$-subacts $D, \varphi(D), \cdots \cup \varphi^{j_{1}-1}(D)$, and each component is a strongly connected $S$-subact.

Case 3. We can proceed as in Case 2, but now all the intersections contain the sink $\{0\}$ and so for any $S$-subact $C$ of at least two elements and for

$$
D=C \cap \varphi^{j_{1}}(C) \cap \cdots \cap \varphi^{j_{r}}(C)
$$

the $(S, \varphi)$-subact $B$ is the 0 -disjoint union of the $S$-subacts $D, \varphi(D), \ldots, \varphi^{j_{1}-1}(D)$ and $D=S$ a for every element $a \in D \backslash\{0\}$.

Case 4. Now $\chi_{\varphi}$ is the Rees congruence determined by $B$.

\section{ACKNOWLEDGEMENT}

Sincere thanks are due to Professor H. E. Heatherly for his kind advise and encouragement.

\section{REFERENCES}

[1] Heatherly, H. E., Lee, E. K. S., and Wiegandt, R.: Involutions on universal algebras, in: Saad, G. and Thomsen, M. J. (Eds.), Nearrings, Nearfields and K-Loops, Proc. conference on nearrings and nearfields (Hamburg, Germany, July 30-August 6, 1995), Kluwer AP, Dordrecht, 1997, pp. 269280

[2] Kilp, M., Knauer, U., and Mikhalev, A. V.: Monoids, Acts and Categories, Walter de Gruyter, Berlin, 2000.

\section{Authors' addresses}

\section{N. V. Loi:}

A. Rényi Institute of Mathematics, P. O. Box 127, H-1364 Budapest, Hungary

E-mail address: nvloi@hotmail.com 


\section{R. Wiegandt:}

A. Rényi Institute of Mathematics, P. O. Box 127, H-1364 Budapest, Hungary

E-mail address: wiegandt@renyi .hu 